\title{
Screening for Atrial Fibrillation Using Smartphones and Smartwatches
}

Haran Burri

Cardiology Department, University Hospital of Geneva, Geneva, Switzerland monitor our patients' health. This article provides an overview on how they may be used to screen for atrial fibrillation, and discusses the current status of this technology.

\section{Keywords}

Atrial fibrillation, screening, diagnosis, photoplethysmography, application, smartphone, smartwatch

Disclosure: Haran Burri has nothing to declare in relation to this article.

Review Process: This article is a short opinion piece and has not been submitted to external peer reviewers.

Authorship: All named authors meet the International Committee of Medical Journal Editors (ICMJE) criteria for authorship of this manuscript, take responsibility for the integrity of the work as a whole, and have given final approval to the version to be published.

open Access: This article is published under the Creative Commons Attribution Noncommercial License which permits any non-commercial use, distribution, adaptation and reproduction provided the original author(s) and source are given appropriate credit. (c) The Author 2018.

Received: 6 September 2017

Published Online: 29 January 2018

Citation: European Journal of Arrhythmia \& Electrophysiology. 2018;4(1):11-13

Corresponding Author: Haran Burri, Cardiology Department, University Hospital of Geneva, Rue Gabrielle Perret Gentil 4, 1211 Geneva 14, Switzerland. E: haran.burri@hcuge.ch

Support: No funding was received in the publication of this article.
Undiagnosed atrial fibrillation (AF) is common, especially in older populations. The European Society of Cardiology (ESC) therefore recommends in its 2016 guidelines' that AF should be screened in all patients $>65$ years old by taking their pulse and, if irregular, diagnosed by recording an electrocardiogram (ECG). Such measures detect previously unknown AF in 1-2\% of these elderly patients, ${ }^{2}$ and the prevalence is likely to be significantly higher if more frequent or continuous monitoring is used. There has therefore been growing interest in developing tools to screen for AF. The interest is shared by healthcare professionals, patients, healthcare payers, and the industry (both pharmaceutical and device manufacturers). The ideal tool would:

- provide continuous monitoring;

- have $100 \%$ accuracy to avoid unnecessary patient stress or false reassurance, as well as increased physician workload;

- be noninvasive;

- be user friendly (to encourage patient adherence);

- give instant feedback to the user to inform them of the status of their rhythm;

- record data that can be easily transmitted for processing and documentation, and;

- be affordable.

However, as of today and for the foreseeable future, no tool entirely satisfies this wish list.

Smartphones and smartwatches are ideal platforms for providing AF screening, as they are ubiquitous, have sensors that can record the pulse, are connected to upload data for offline processing and storage, are able to provide feedback to the patient and limit costs because usually no extras (other than apps) are required.

The signals that can be used by smartphones for recording cardiac rhythm are the ECG (using add-on hardware), seismocardiography (SCG) and photoplethysmography (PPG). single-lead ECG signals are recorded by electrodes, which can be applied to the smartphone casing or integrated into the smartwatch wristband (AliveCor, San Francisco, CA), which then transmits data wirelessly to the smartphone or smartwatch. This technology has been validated for AF detection by a number of publications, showing up to $98 \%$ sensitivity and $97 \%$ specificity, ${ }^{3}$ with a lower sensitivity of $55 \%$ in a recent publication. ${ }^{4}$ Other handheld ECG recorders, such as the Beurer ME 90 (UIm, Germany), can connect wirelessly to smartphones for real-time and offline visualisation of the ECG tracing, but the performance of the automatic arrhythmia detection algorithm has not as yet been validated. SCG records cardiac rhythm using the smartphone accelerometer and gyroscope. ${ }^{5}$ The smartphone is placed on the chest while the patient is recumbent, which is impractical and limits use of this technique. Pulse is more readily measured using PPG. The patient places their finger on the smartphone camera, which measures pulsatile bloodflow (lit up by the flash). Some smartwatches are equipped with green LED lights paired with photodiodes on the back of the casing to measure the PPG signal. Another investigational method is analysis of the contact-free facial pulsatile PPG signals using the smartphone camera. ${ }^{6}$ 
Figure 1: Recording of the author's pulse with 5 minute finger photoplethysmography using a smartphone and the Heartbeats app (Preventicus, Jena, Germany)

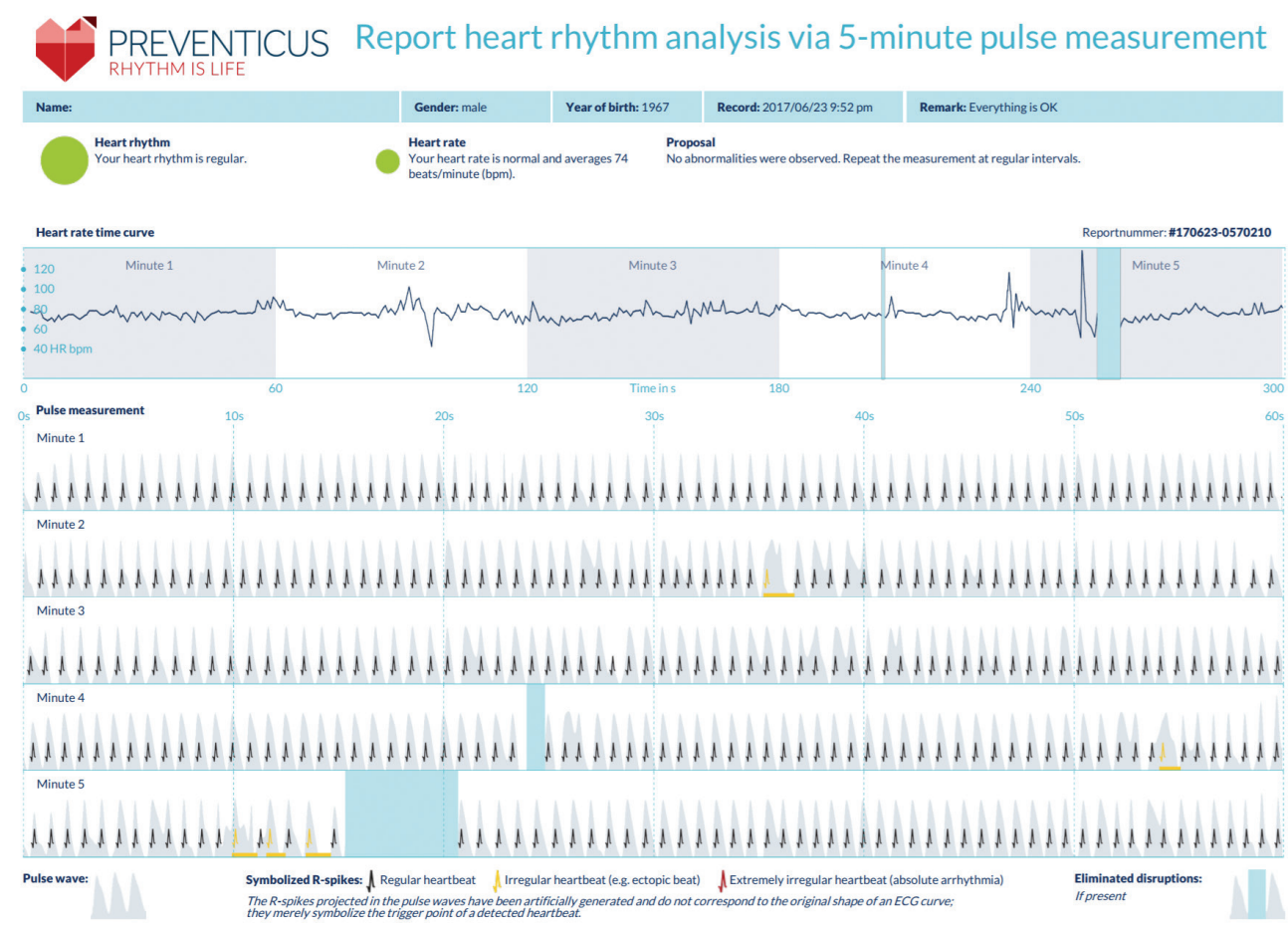

The rhythm was classified as 'normal'. Note the blanked segments (not taken into account for analysis) designated by the blue rectangles, where motion artefacts (created intentionally) disrupted the signals.

Figure 2: Finger photoplethysmography recording in a 74-year-old male patient with chronic atrial fibrillation using a smartphone and the Heartbeats app (Preventicus, Jena, Germany)

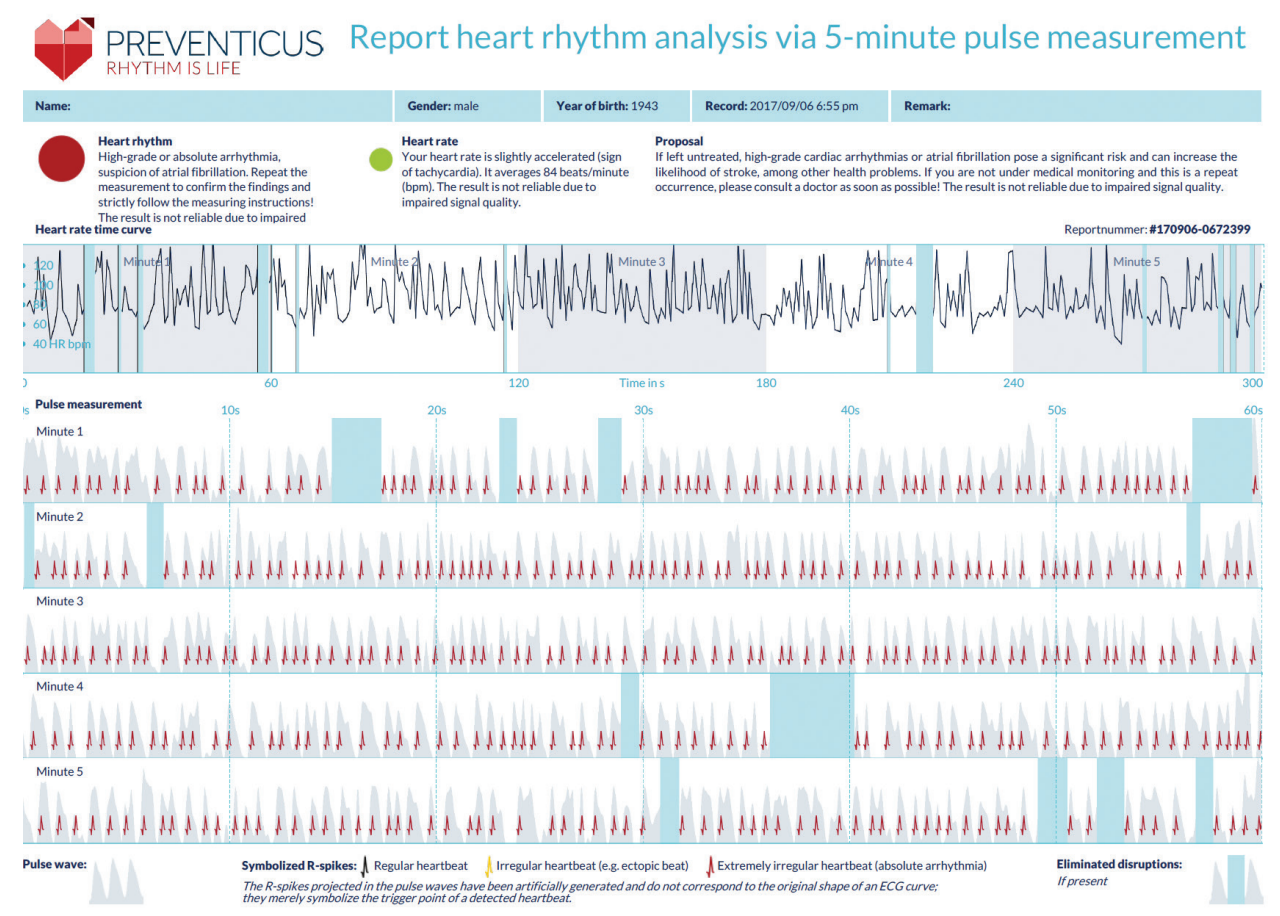

The rhythm was classified as arrhythmia with suspected atrial fibrillation.

A large number of free apps to measure heart rate using PPG signals are available, but those that process data for AF screening are limited. Screening of AF using smartphone PPG was first reported by Lee et al. in 2012. AF may be identified by assessing variability in RR intervals using mathematical analyses such as the root mean square of successive difference (RMSSD), Shannon Entropy (ShE) and Poincarré plots, either alone or in combination, yielding $96-97 \%$ sensitivity and $94-98 \%$ specificity for diagnosing AF. ${ }^{8.9}$ Other strategies involve self-similarity 
of waveforms, ${ }^{9}$ or artificial intelligence (using a 'deep neural network') which has recently been shown to detect AF with $98 \%$ sensitivity and $90 \%$ specificity in a study involving 51 patients who wore an Apple watch for 20 minutes before and after electrical cardioversion for $A F^{10}$

There are a number of commercially available apps in Europe to screen for AF. The Photo AFib Detector (CCAPP, Hong Kong, China) and AF Detect (HealthSTATS, London, UK) can be downloaded by the general public for $<€ 2$, but have never been clinically validated. Both have legal disclaimers stating that they are not for medical use. As with any diagnostic test, validation of accuracy is mandatory, and the method should be subjected to regulatory approval before being used in clinical practice. Two apps, listed below, have so far received conformité Européene (CE) approval.

Fibricheck (Qopium, Hasselt, Belgium) has been clinically validated for regulatory purposes (publications are pending) and is for the time being only being prescribed by physicians. The AF detection algorithm uses artificial intelligence with signal pattern recognition and offline analysis, requiring an internet connection. The patient receives immediate feedback on their pulse rate and normal/abnormal heart rhythm following 1 minute pulse acquisition by his or her smartphone. The recordings are processed by a teleservice centre for verification, with a full report then being sent to the physician.

Heartbeats (Preventicus, Jena, Germany) can be downloaded directly by the general public and provides immediate feedback to the patient (data analysis is performed offline, and requires an internet connection). Examples of recordings are shown in Figures 1 and 2. Unlike conventional methods for determining pulse rate by simply using signal peaks, a combination of detection of the steepest slope of each pulse wave and a correlation method with a pulse wave pattern is used. This proprietary algorithm was validated and yielded an excellent correlation of $r=0.99$, compared with RR intervals from standard ECG recordings." For AF screening, the tachogram is filtered to eliminate premature beats and other disruptions using an algorithm for adaptive variance estimation. ${ }^{12}$ AF is evaluated using RMSSD, Shanon entropy and Poincarré plots. Using 2 minute recordings, sensitivity and specificity for diagnosing AF were 87.5 and $95 \%$, respectively, using either RMSSD alone or in combination with Shannon entropy. Sensitivity and was slightly higher (95\%) with the 5 minute recordings using RMSSD and Poincarre analysis, with the same specificity (95\%). Additional data analysis by a service centre is available, which is likely to enhance specificity. Results using the algorithm with signals from a smartwatch have recently been presented in abstract form, ${ }^{13}$ and showed $85 \%$ sensitivity and $82 \%$ specificity, with 25/109 (23\%) patients who had unsuitable signals. This is a first step, and a more recent version of the proprietary algorithm incorporates data from the accelerometer of the mobile device, which is believed to improve rejection of motion artefacts and improve diagnostic accuracy in studies which are currently underway.

The PPG signal from smartphones and smartwatches are currently unable per se to provide data of diagnostic quality. This technology is therefore more suited for screening purposes and will require additional testing to diagnose AF. The challenges that physicians will be faced with include interpretation of PPG signals, deciding which patients should be further evaluated and which confirmatory tests to perform. Implantable loop recorders or ECG patches ${ }^{14}$ are options, but both solutions come at a cost which will require economic assessment. Another strategy would be to use a two-step process, first with PPG signals for continuous monitoring (with a smartwatch or wristband). In case of suspected $A F$, a signal would be triggered to alert the patient to record an ECG using a handheld device (e.g. an AliveCor or Beurer recorder). This second step would provide further automatic analysis (this time of the ECG) to determine whether AF is present, and could be later reviewed by a physician if positive, to diagnose or rule out arrhythmia.

There is legitimate concern that AF screening by smartphones and smartwatches may cause increased workload and responsibility for physicians. ${ }^{15}$ It may also cause undue stress to patients. It is therefore mandatory that this technology be carefully validated in clinical studies which reflect ambulatory use in a primary care setting (rather than, for example, laboratory testing in immobile patients before and after AF cardioversion).

\section{Conclusion}

In addition to rhythm monitoring, PPG may in future have additional uses that can be integrated into smartphone and smartwatch apps, such as monitoring oxygen saturation and blood pressure, or screening for sleep apnoea. The arterial pulse has been palpated since ancient times to evaluate disease states. ${ }^{16}$ PPG has modernised the analysis of this physiological signal. In the era of multifunctional smartphones and smartwatches, it is very likely that PPG will be increasingly used to empower the general public to manage their own healthcare. $\square$
1. Kirchhof P, Benussi S, Kotecha D, et al. 2016 ESC Guidelines for the management of atrial fibrillation developed in collaboration with EACTS. Eur Heart J. 2016;37:2893-962.

2. Lowres N, Neubeck L, Redfern J, Freedman SB. Screening to identify unknown atrial fibrillation. A systematic review. Thromb Haemost. 2013:110:213-22.

3. Lau JK, Lowres N, Neubeck L, et al. iPhone ECG application for community screening to detect silent atrial fibrillation: a nove technology to prevent stroke. Int J Cardiol. 2013;165:193-4.

4. Desteghe L, Raymaekers Z, Lutin M, et al. Performance of handheld electrocardiogram devices to detect atrial fibrillation in a cardiology and geriatric ward setting. Europace. 2017;19:29-39.

5. Lahdenoja O, Hurnanen T, Iftikhar Z, et al. Atrial fibrillation detection via accelerometer and gyroscope of a smartphone. IEEE Journal of Biomedical and Health Informatics. 2017;99:1.

6. Yan BP, Chan S, Lai WH, et al. Abstract 17351: Validation of a novel contact-free atrial fibrillation screening method using an Iphone camera to detect facial pulsatile photoplethysmographic signals. Circulation. 2016;134:A17351-A17351

7. Lee J, Reyes $B A, M C M a n u s ~ D D$, et al. Atrial fibrillation detection using a smart phone. Conf Proc IEEE Eng Med Biol SOC. 2012;1177-80.

8. MCManus DD, Lee J, Maitas $\mathrm{O}$, et al. A novel application for the detection of an irregular pulse using an iPhone 4 S in patients detection of an irregular pulse using an iPhone 4 S in
with atrial fibrillation. Heart Rhythm. 2013;10:315-9.

9. Chan $\mathrm{PH}$, Wong $\mathrm{CK}$, Poh $\mathrm{YC}$, et al. Diagnostic performance of a smartphone-based photoplethysmographic application for atrial fibrillation screening in a primary care setting. J Am Heart AssOC. 2016;5.

10. Sanchez JM, Ballinger B, Olgin JE, et al. AF detection and ablation outcomes: Answering questions that matter to patients: Detecting atrial fibrillation using a smart watch - the mRhythm study. Presented at: HRS Heart Rhythm, Chicago, IL, mRhythm study. Presented at:
11. Koenig N, Seeck A, Eckstein J, et al. Validation of a new heart Rate measurement algorithm for fingertip recording of video signals with smartphones. Telemed J E Health. 2016;22:631-6.

12. Voss A, Heitmann A, Schroeder R, et al. Short-term heart rate variability--age dependence in healthy subjects. Physiol Meas. 2012;33:1289-311.

13. Djurdjevic A, Stöckli R, Rhinisperger M, et al. WATCH AF SmartWATCHes for detection of Atrial Fibrillation. Presented at: Europace, Vienna, Austria, 18 June 2017, Abstr \#P287.

14. Barrett PM, Komatireddy R, Haaser S, et al. Comparison of 24-hour Holter monitoring with 14-day novel adhesive patch electrocardiographic monitoring. Am J Med. 2014;127:95.e11-97.

15. Callans DJ, I left my smartphone at home and can't tell if I'm in atrial fibrillation. Heart Rhythm. 2013;10:320-1.

16. Ghasemzadeh N, Zafari AM, A brief journey into the history of the arterial pulse. Cardiol Res Pract. 2011;2011:14. 